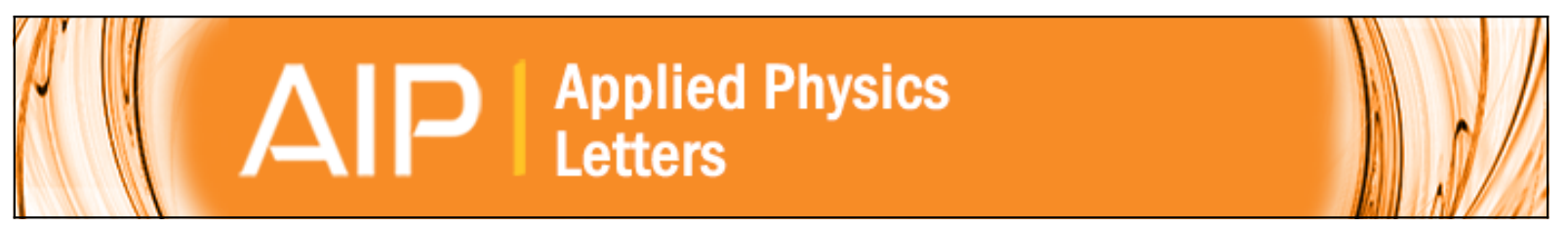

\title{
Enhanced overall efficiency of GalnN-based light-emitting diodes with reduced efficiency droop by Al-composition-graded AIGaN/GaN superlattice electron blocking layer
}

Jun Hyuk Park, Dong Yeong Kim, Sunyong Hwang, David Meyaard, E. Fred Schubert, Yu Dae Han, Joo Won Choi, Jaehee Cho, and Jong Kyu Kim

Citation: Applied Physics Letters 103, 061104 (2013); doi: 10.1063/1.4817800

View online: http://dx.doi.org/10.1063/1.4817800

View Table of Contents: http://scitation.aip.org/content/aip/journal/apl/103/6?ver=pdfcov

Published by the AIP Publishing

\section{Articles you may be interested in}

Enhancing the performance of green $\mathrm{GaN}$-based light-emitting diodes with graded superlattice AIGaN/GaN inserting layer

Appl. Phys. Lett. 103, 102104 (2013); 10.1063/1.4819879

Publisher's Note: "Performance enhancement of blue light-emitting diodes with a special designed AIGaN/GaN superlattice electron-blocking layer" [Appl. Phys. Lett. 99, 221103 (2011)]

Appl. Phys. Lett. 102, 089901 (2013); 10.1063/1.4752472

The reduction of efficiency droop by Al0.82 $\mathrm{In} 0.18 \mathrm{~N} / \mathrm{GaN}$ superlattice electron blocking layer in (0001) oriented GaN-based light emitting diodes

Appl. Phys. Lett. 101, 131113 (2012); 10.1063/1.4756791

Performance enhancement of blue light-emitting diodes with a special designed AlGaN/GaN superlattice electron-blocking layer

Appl. Phys. Lett. 99, 221103 (2011); 10.1063/1.3653390

Stable temperature characteristics of InGaN blue light emitting diodes using AIGaN/GaN/InGaN superlattices as electron blocking layer

Appl. Phys. Lett. 96, 091104 (2010); 10.1063/1.3340939

\section{Want to publish your paper in the \#1 MOST CITED journal in applied physics?}

\author{
With Applied Physics Letters, you can.
}

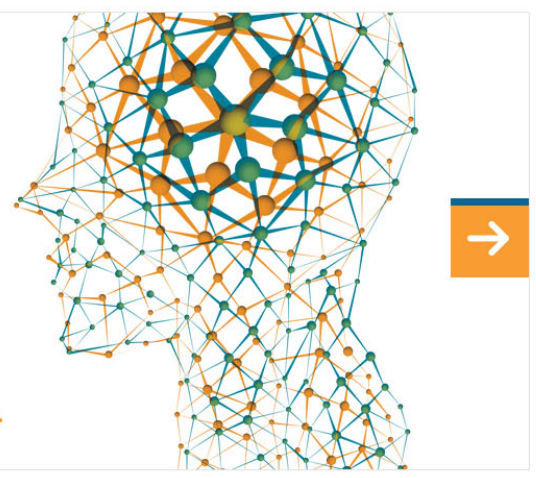




\title{
Enhanced overall efficiency of GalnN-based light-emitting diodes with reduced efficiency droop by Al-composition-graded AIGaN/GaN superlattice electron blocking layer
}

\author{
Jun Hyuk Park, ${ }^{1}$ Dong Yeong Kim, ${ }^{1}$ Sunyong Hwang, ${ }^{1}$ David Meyaard, ${ }^{2}$ E. Fred Schubert, ${ }^{2}$ \\ Yu Dae Han, ${ }^{3}$ Joo Won Choi, ${ }^{3}$ Jaehee Cho, $^{4}$ and Jong Kyu Kim ${ }^{1, a)}$ \\ ${ }^{1}$ Department of Materials Science and Engineering, Pohang University of Science and Technology (POSTECH), \\ Pohang 790-784, South Korea \\ ${ }^{2}$ Future Chips Constellation, Department of Electrical, Computer, and Systems Engineering, \\ Rensselaer Polytechnic Institute, Troy, New York 12180, USA \\ ${ }^{3}$ Seoul Opto-device Co., 725-5 Wonsi-dong, Danwon-gu, Ansan City, Kyunggi-do 425-851, South Korea \\ ${ }^{4}$ School of Semiconductor and Chemical Engineering, Semiconductor Physics Research Center, \\ Chonbuk National University, Jeonju 561-756, South Korea
}

(Received 7 July 2013; accepted 22 July 2013; published online 5 August 2013)

\begin{abstract}
$\mathrm{Al}_{x} \mathrm{Ga}_{1-x} \mathrm{~N} / \mathrm{GaN}$ superlattice electron blocking layers (EBLs) with gradually decreasing $\mathrm{Al}$ composition toward the p-type GaN layer are introduced to GaInN-based high-power light-emitting diodes (LEDs). GaInN/GaN multiple quantum well LEDs with 5- and 9-period Al-compositiongraded $\mathrm{Al}_{x} \mathrm{Ga}_{1-x} \mathrm{~N} / \mathrm{GaN}$ EBL show comparable operating voltage, higher efficiency as well as less efficiency droop than LEDs having conventional bulk AlGaN EBL, which is attributed to the superlattice doping effect, enhanced hole injection into the active region, and reduced potential drop in the EBL by grading $\mathrm{Al}$ compositions. Simulation results reveal a reduction in electron leakage for the superlattice EBL, in agreement with experimental results.

(C) 2013 AIP Publishing LLC. [http://dx.doi.org/10.1063/1.4817800]
\end{abstract}

GaN-based light-emitting diodes (LEDs) have attracted considerable attention as energy-efficient and environmentally benign solid-state lighting sources. However, the gradual decrease in internal quantum efficiency (IQE) with increasing input current, called efficiency droop, is a major obstacle in the widespread adoption of LEDs for general illumination. Many physical mechanisms including Auger recombination, ${ }^{1,2}$ delocalization of carriers from In-rich low-defectdensity regions, ${ }^{3,4}$ and electron leakage ${ }^{5,6}$ have been proposed as the possible reasons for efficiency droop. Recently, Meyaard et al. reported that the asymmetry in carrier transport, caused by much lower concentration and mobility of holes, compared with electrons, is the dominant mechanism causing for efficiency droop. ${ }^{7,8}$ Introducing a highly p-doped AlGaN electron-blocking layer (EBL) with a high Al composition may mitigate the degree of the asymmetry by means of a high potential barrier for electron leakage but a low barrier for hole injection. However, it is very difficult to realize such an ideal EBL because as the $\mathrm{Al}$ composition in $\mathrm{AlGaN}$ increases (i) the hole concentration decreases due to increase in the ionization energy of the p-type dopant $\mathrm{Mg}$, and (ii) the potential barrier for blocking electron leakage is counteracted by the increase in positive piezoelectric polarization sheet charge at the interface between the GaN spacer and the AlGaN EBL. Mg doped AlGaN/GaN superlattices (SLs) have been introduced to increase the hole concentration and to block electrons escaping from the active region; ${ }^{9-14}$ however, such SL EBLs cause a penalty in operating voltage, due to hole transport that is hindered by the series of potential barriers at the $\mathrm{AlGaN} / \mathrm{GaN}$ hetero-interfaces of the SL EBL.

\footnotetext{
${ }^{\text {a) }}$ Author to whom correspondence should be addressed. Electronic mail: kimjk@postech.ac.kr.
}

In this study, we present AlGaN/GaN SL-EBLs having a graded Al mole fraction, called graded SL-EBL (GSL-EBL), designed for achieving, simultaneously, a high IQE and reduced efficiency droop while avoiding the operating voltage penalty. In agreement with our simulation results, GaInN/GaN MQW LEDs with AlGaN/GaN GSL-EBLs show both higher IQE and reduced efficiency droop, compared to LEDs with conventional bulk AlGaN EBL.

The optoelectronic properties of GaInN/GaN MQW LEDs $\left(1 \times 1 \mathrm{~mm}^{2}\right)$ with various EBL structures - conventional bulk $\mathrm{Al}_{0.13} \mathrm{Ga}_{0.87} \mathrm{~N}$ EBL, 9-period $\mathrm{Al}_{0.23} \mathrm{Ga}_{0.77} \mathrm{~N} / \mathrm{GaN}$ SLEBL, and 9-period $\mathrm{Al}_{x} \mathrm{Ga}_{1-x} \mathrm{~N} / \mathrm{GaN}$ GSL-EBL ( $x$ varies from 0.23 to 0.15 ), as schematically shown in Fig. 1were simulated by using the Advance Physical Model of Semiconductor Devices (APSYS) simulator. Commonly accepted physical parameters were used in the simulations, including Auger coefficient of $1 \times 10^{-31} \mathrm{~cm}^{6} / \mathrm{s}$, radiative recombination coefficient of $10^{-11} \mathrm{~cm}^{3} / \mathrm{s}$, spontaneous and piezoelectric polarization elementary charge densities of $1.04 \times 10^{13}$ and $2.74 \times 10^{12} \mathrm{~cm}^{-2}$ in the MQW and the EBL, respectively. ${ }^{15}$ Band parameters for ternary layers, such as GaInN or AlGaN were calculated by using Vegard's rule while considering the bowing effect. Figures 2(a) and 2(b) show calculated energy band diagrams, the hole concentration in each quantum well, and the electron current density at the forward current density of $35 \mathrm{~A} / \mathrm{cm}^{2}$. The polarization mismatch at hetero-interfaces results in the formation of large sheet charges, causing large triangularly shaped $\mathrm{GaN}$ barriers between the GaInN MQWs. For the reference LED with bulk $\mathrm{Al}_{0.13} \mathrm{Ga}_{0.87} \mathrm{~N} \mathrm{EBL}$, the positive polarization sheet charge at the interface between the $\mathrm{GaN}$ spacer and the EBL pulls down the conduction band edge, and thus, lowers the effective potential barrier $\left(\Delta E_{\mathrm{C}}\right)$ 


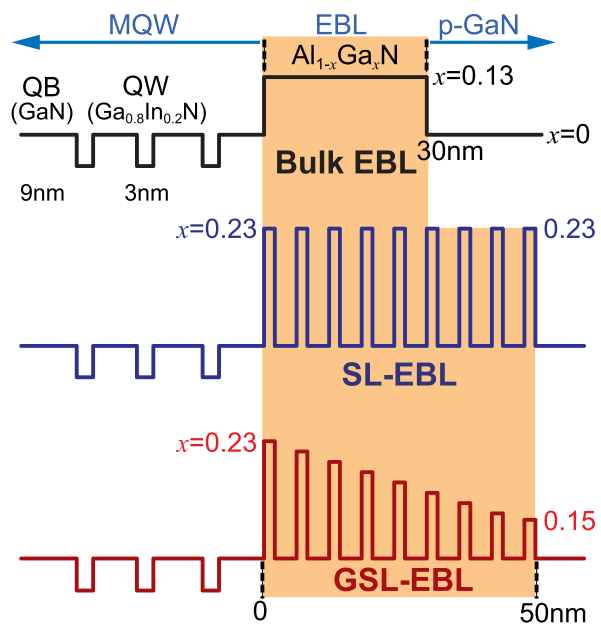

FIG. 1. Schematic conduction band diagrams showing the three different EBL structures-the conventional bulk $\mathrm{Al}_{0.13} \mathrm{Ga}_{0.87} \mathrm{~N}$ EBL, a 9-period $\mathrm{Al}_{0.23} \mathrm{Ga}_{0.77} \mathrm{~N} / \mathrm{GaN}$ SL-EBL, and a 9-period $\mathrm{Al}_{x} \mathrm{Ga}_{1-\mathrm{x}} \mathrm{N} / \mathrm{GaN}$ GSL-EBL ( $x$ varies from 0.23 to 0.15 ).

intended to prevent electron leakage. Increasing the $\mathrm{Al}$ composition in the EBL increases $\Delta E_{\mathrm{C}}$ but pulls the conduction band further down, thereby preventing an effective suppression of the electron leakage. Consequently, the

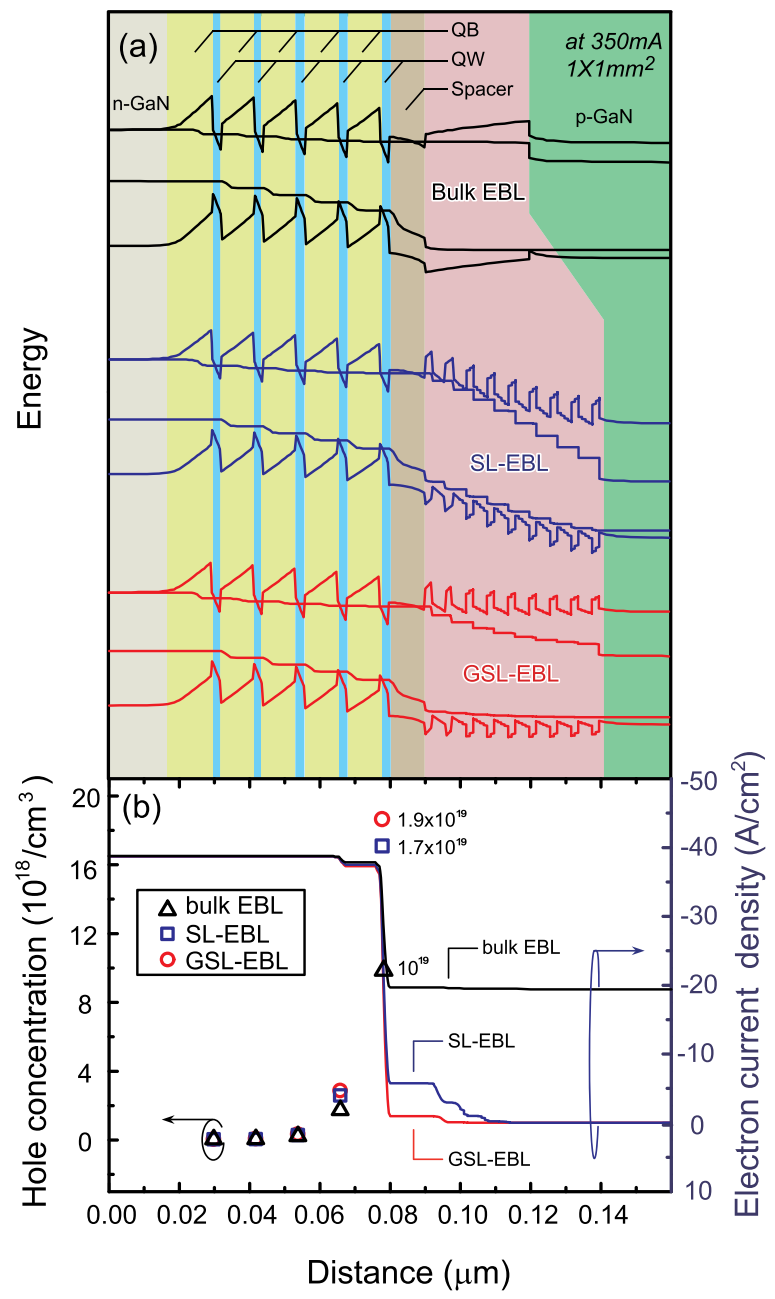

FIG. 2. (a) The energy band diagrams and (b) the hole concentration and electron leakage current of the GaInN/GaN MQW LEDs with three different EBL structures at an injection current level of $350 \mathrm{~mA}$ calculated by APSYS simulator. electron current density that escapes from the MQW active region-electron leakage-is very high, which may cause the significant efficiency droop and poor IQE.

The electron leakage is remarkably reduced by using SL-EBL and GSL-EBL because the SL-doping effect ${ }^{9}$ together with higher $\mathrm{Al}$ composition in $\mathrm{AlGaN}$ result in more effective electron blocking as well as better hole injection than the bulk EBL case, as shown in Fig. 2(b). However, the potential drop in the SL-EBL region is severe, as shown in the energy band diagram, indicating that the SL-EBL LED suffers from a high operating voltage despite the SL doping effect. This is attributed to the large lattice mismatch at the $\mathrm{AlGaN} / \mathrm{GaN}$ hetero-interfaces causing large polarization-induced electric fields, as well as to the higher overall Al composition of the SL-EBL compared with the bulk EBL. By grading the Al composition, the voltage drop across the GSL-EBL becomes smaller than that for the SL-EBL due to the smaller lattice mismatch between AlGaN and GaN layer and lower overall Al content in the EBL. In addition, the lower Al composition on the p-type side reduces the potential barrier for hole injection, leading to less electron leakage and a higher hole concentration at the last-grown quantum well where most of the radiative recombination occurs. ${ }^{16}$ As expected from the calculated energy band diagram and from the distribution of carriers under steady-state operation, the LED with GSL-EBL shows the highest peak efficiency and lowest efficiency droop. The operating voltage for the GSL-EBL LED is lower than the SL-EBL LED, however higher than that for the reference LED, which we attribute to the much larger proportion of resistive AlGaN, which can be overcome by using a GSL-EBL with fewer periods, for example, a 5-period GSL-EBL.

For experimental verification of the advantageous effect of the GSL-EBL, GaInN/GaN MQW LEDs with three different EBL structures-conventional bulk AlGaN EBL, 9-period $\mathrm{Al}_{x} \mathrm{Ga}_{1-x} \mathrm{~N} / \mathrm{GaN}$ GSL-EBL (9-period GSL-EBL), and 5-period $\mathrm{Al}_{x} \mathrm{Ga}_{1-x} \mathrm{~N} / \mathrm{GaN}$ GSL-EBL (5-period GSLEBL) - were grown on c-plane sapphire substrate by metalorganic vapor phase epitaxy. A $2 \mu$ m-thick unintentionally doped GaN was grown first, followed by a 20-period of GaInN/GaN short-period SL layer, a $3 \mu$ m-thick Si-doped n-type $\mathrm{GaN}$ layer $\left(\mathrm{n}=5 \times 10^{18} \mathrm{~cm}^{-3}\right)$, and a 5-period $\mathrm{Ga}_{0.8} \mathrm{In}_{0.2} \mathrm{~N}$ (3 nm, undoped)/GaN (9 nm, Si-doped, $\mathrm{n}=5$ $\times 10^{17} \mathrm{~cm}^{-3}$ ) MQW active region. The three different types of EBL structures were grown on top of the MQW active region. The $18 \mathrm{~nm}$-thick p-type $\mathrm{Al}_{0.20} \mathrm{Ga}_{0.80} \mathrm{~N}$ bulk EBL structure was used as a reference. The 5-period GSL-EBL consisted of 5 periods of $\mathrm{Al}_{x} \mathrm{Ga}_{1-x} \mathrm{~N} / \mathrm{GaN}$ bi-layers ( $x$ varies from 0.23 to 0.07 with 0.04 intervals) with thicknesses of $2 \mathrm{~nm}$ for the AlGaN quantum barriers (QBs) and $3.6 \mathrm{~nm}$ for GaN quantum wells (QWs). Likewise, the 9-period GSLEBL consisted of 9 periods of $\mathrm{Al}_{x} \mathrm{Ga}_{1-x} \mathrm{~N} / \mathrm{GaN}$ bi-layers ( $x$ varies from 0.23 to 0.07 with 0.02 intervals) with thickness of $2 \mathrm{~nm}$ for both QWs and QBs, respectively. Both AlGaN and GaN in the GSL-EBLs are $\mathrm{Mg}$ doped to realize the SL-doping effect. Finally, a $100 \mathrm{~nm}$-thick $\mathrm{Mg}$ doped p-type GaN layer $\left(p=4 \times 10^{17} \mathrm{~cm}^{-3}\right)$ was grown on the EBLs. The three LED wafers having the 3 different EBLs were processed into $600 \times 600 \mu \mathrm{m}^{2}$ lateral-structure devices 
in the same processing run. The LED mesa structures were obtained by photolithographic patterning followed by inductively coupled plasma reactive-ion etching with $\mathrm{BCl}_{3} / \mathrm{Cl}_{2}$ gas. Subsequently, Ti/Al n-type ohmic contacts were deposited on the exposed n-type GaN by electron-beam evaporation and annealed at $650^{\circ} \mathrm{C}$ for $1 \mathrm{~min}$ in $\mathrm{N}_{2}$ ambient. ITO p-type ohmic contacts were deposited on the mesa and annealed at $500{ }^{\circ} \mathrm{C}$ for $1 \mathrm{~min}$ in air ambient, followed by the deposition of $\mathrm{Cr} / \mathrm{Au}$ pad metal.

The grading of $\mathrm{Al}$ composition in the EBLs was examined by using high angle annular dark field (HAADF) scanning transmission electron microscopy (STEM). Figure 3 shows a schematic LED structure and the corresponding HAADF STEM image. Enlarged HAADF-STEM images show the intensity profiles of the three different EBL structures. Since the HAADF intensity is approximately proportional to the square of atomic number, darker regions represent the $\mathrm{AlGaN}$ while the brighter regions represent the GaN. HAADF intensity of AlGaN decreases gradually from the MQW active region to the p-type GaN layer, indicating that the $\mathrm{Al}$ composition was properly graded.

Light output-current-voltage (L-I-V) characteristics of the LEDs were measured by using a Keithley 2520 pulsed laser diode test system with $10 \mu$ s pulse-duration and a $1 \%$ duty cycle. Almost identical L-I-V characteristics are obtained for each type of device. Figure 4 shows the representative external quantum efficiency (EQE) as a function of current density, and the inset shows the I-V characteristics. The LEDs with 9-period GSL-EBL show a higher EQE throughout the whole injection current density range as well as a lower efficiency droop than that of the reference LEDs. The efficiency droop at $100 \mathrm{~mA}$ is measured to be $7.8 \%$ and 9.7\% for the 9-period GSL-EBL and the reference LEDs, respectively. However, at $20 \mathrm{~mA}$, the operating voltage for the 9-period GSL-EBL LED is $3.05 \mathrm{~V}$, slightly higher than that of the reference LED $(3.00 \mathrm{~V})$, possibly due to the thicker and more resistive AlGaN layer in the 9-period GSLEBL. The LEDs with a reduced number of periods of the GSL, i.e., the 5-period GSL-EBL, show the lowest operating voltage of $2.95 \mathrm{~V}$ as expected, and a much reduced efficiency droop of $5.6 \%$ but also the lowest peak efficiency.
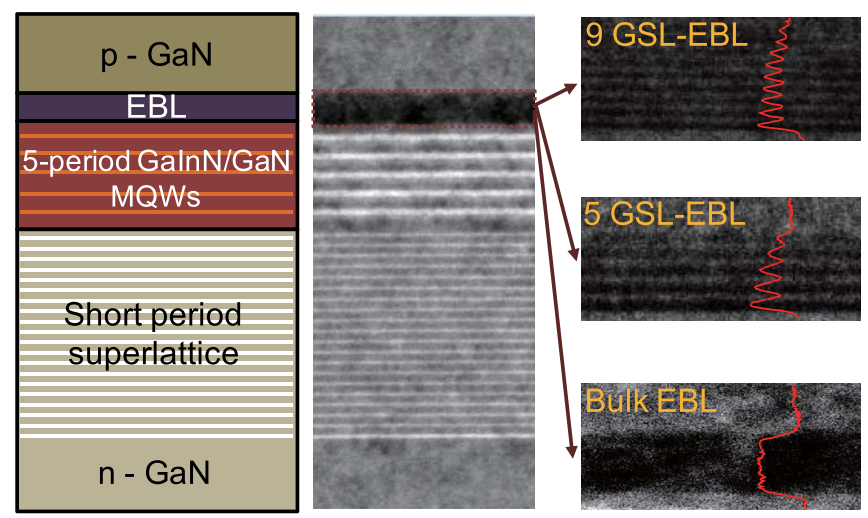

FIG. 3. Schematic LED structure together with the corresponding HAADF STEM image. Enlarged HADDF STEM images are 9-period GSL-EBL, 5-period GSL-EBL, and reference bulk EBL. GSL EBLs show gradual decrease in $\mathrm{Al}$ composition from the MQW active region to the p-type $\mathrm{GaN}$ layer.

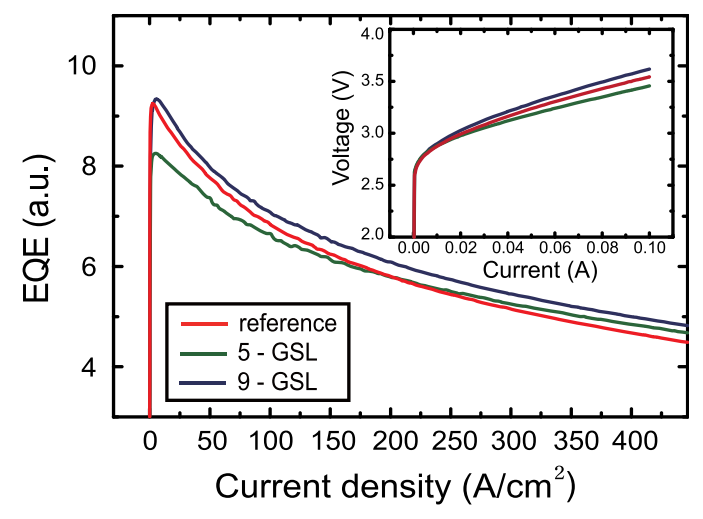

FIG. 4. EQE as a function of injection current for the GaInN LEDs with three different EBL structures. The inset shows the I-V characteristics.

In order to investigate the cause of the low peak efficiency for the 5-period GSL-EBL LED, temperaturedependent L-I-V characteristics were measured and analyzed by using the $A B+f(n)$ model $^{17-19}$ where $A$ and $B$ represent the Shockley-Read-Hall (SRH) and radiative recombination coefficients, respectively, and the $f(n)$ represents the droop causing term consisting of Auger coefficient and carrier leakage term. By considering this model, it is possible to estimate the relative contribution of $A, B$, and $f(n)$ coefficients to the total recombination. Figure 5 shows temperaturedependency of normalized light output power (LOP) and the contribution of SRH recombination extracted from the $A B+f(n)$ analyses at the relatively low current density of $5 \mathrm{~A} / \mathrm{cm}^{2}$ for temperatures ranging from $298 \mathrm{~K}$ to $473 \mathrm{~K}$. It is well known that the SRH non-radiative recombination plays a relatively large role at low current densities and its lifetime decreases with increasing temperature, leading to the decrease in LOP and the increase in the fraction of SRH recombination for all samples as the temperature increases. ${ }^{17,20}$ Note that the LEDs with 5-period GSL-EBL show a much more rapid increase in LOP and decrease in SRH as the temperature increases than the other LEDs, indicating that the SRH recombination is predominant in the LEDs with 5-period GSL-EBL. We believe that the crystal quality of the LEDs with 5-period GSL-EBL is somewhat inferior to that of other LEDs, causing the increased SRH recombination, thus lowering the peak efficiency at low current densities as shown in Fig. 4. At high current densities,

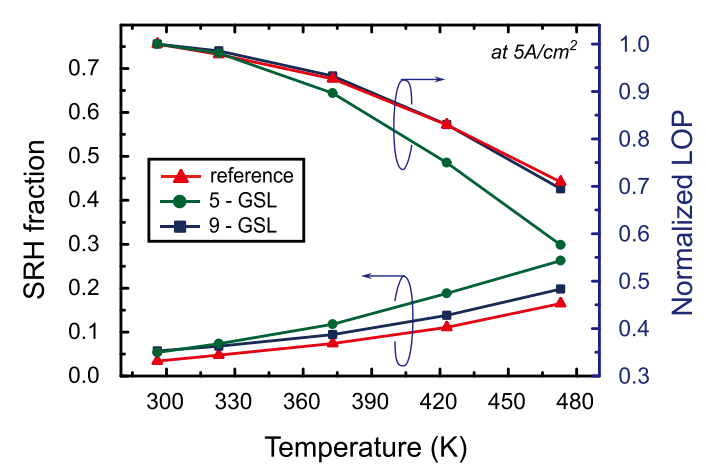

FIG. 5. The Shockley-Read-Hall (SRH) fraction of the total recombination as estimated from the $A B+f(n)$ model fit and normalized light output power (LOP) as a function of temperature for the reference LED, the 5-period GSL EBL LED, and the 9-period GSL EBL LED. 
however, the effect of SRH recombination becomes negligible compared to other high-current efficiency loss mechanisms including carrier leakage and Auger recombination. As a result, the higher efficiency of the 5period GSL-EBL LEDs was observed above the current density of $200 \mathrm{~A} / \mathrm{cm}^{2}$. We anticipate that GSL-EBL LEDs optimized in terms of crystal quality and structure will show not only a higher efficiency with reduced droop but also an even lower operating voltage than LEDs with conventional bulk EBL.

In conclusion, we present a highly promising structure for high power GaInN-based LEDs with, simultaneously, reduced efficiency droop, enhanced overall efficiency, and comparable operating voltage. The structure includes an EBL composed of a p-doped graded-composition AlGaN/ $\mathrm{GaN}$ superlattice and enables better hole injection and reduces electron leakage. It is experimentally shown that GaInN/GaN MQW LEDs with the GSL-EBL show lower efficiency droop and higher EQE as well as comparable or even lower operating voltage compared to LEDs with conventional AlGaN EBLs.

Authors gratefully acknowledge support by the Industrial Technology Development Program and International Collaborative R\&D Program funded by Korean Ministry of Knowledge Economy, Global Research Network program (2011-220-D00064), and Basic Science Research program through the National Research Foundation of Korea (NRF) funded by the Ministry of Education, Science and Technology of Korea (2010-0006646), and POS-LED Center by POSCO and Seoul Semiconductor.
${ }^{1}$ Y. C. Shen, G. O. Mueller, S. Watanabe, N. F. Gardner, A. Munkholm, and M. R. Krames, Appl. Phys. Lett. 91, 141101 (2007).

${ }^{2}$ J. Iveland, L. Martinelli, J. Peretti, J. S. Speck, and C. Weisbuch, Phys. Rev. Lett. 110, 177406 (2013).

${ }^{3}$ B. Monermar and B. E. Semelius, Appl. Phys. Lett. 91, 181103 (2007).

${ }^{4}$ T. Mukai, M. Yamada, and S. Nakamura, Jpn. J. Appl. Phys., Part 138 , 3976 (1999).

${ }^{5}$ J. Wang, L. Wang, W. Zhao, Z. Hao, and Y. Luo, Appl. Phys. Lett. 97, 201112 (2010).

${ }^{6}$ C. H. Wang, J. R. Chen, C. H. Chiu, H. C. Kuo, Y. L. Li, T. C. Lu, and S. C. Wang, IEEE Photon. Technol. Lett. 22, 236 (2010).

${ }^{7}$ D. S. Meyaard, G. B. Lin, Q. Shan, J. H. Cho, and E. F. Schubert, Appl. Phys. Lett. 99, 251115 (2011).

${ }^{8}$ G. B. Lin, D. Meyaard, J. H. Cho, E. F. Schubert, and H. W. Shim, Appl. Phys. Lett. 100, 161106 (2012).

${ }^{9}$ E. F. Schubert, W. Grieshaber, and I. D. Goepfert, Appl. Phys. Lett. 69, 3737 (1996).

${ }^{10}$ J. K. Kim, E. L. Waldron, Y. L. Li, Th. Gessmann, and E. F. Schubert, Appl. Phys. Lett. 84, 3310 (2004).

${ }^{11}$ I. D. Goepfert, E. F. Schubert, A. Osinsky, P. E. Norris, and N. N. Faleev, J. Appl. Phys. 88, 2030 (2000).

${ }^{12}$ L. Chernyak, A. Osinsky, V. N. Fuflyigin, J. W. Graff, and E. F. Schubert, IEEE Trans. Electron Devices 48, 433 (2001).

${ }^{13}$ S. J. Lee, S. H. Han, C. Y. Cho, S. P. Lee, D. Y. Noh, H. W. Shim, Y. C. Kim, and S. J. Park, J. Phys. D: Appl. Phys. 44, 105101 (2011).

${ }^{14}$ Y. Y. Zhang and Y. A. Yin, Appl. Phys. Lett. 99, 221103 (2011).

${ }^{15}$ F. Bernardini, in Nitride Semiconductor Devices: Principles and Simulation, edited by J. Piprek (Wiley-VCH, Weinheim, 2007), pp. 49-67.

${ }^{16}$ A. David, M. J. Grundmann, J. F. Karding, N. F. Gardner, T. G. Mihopoulos, and M. R. Krames, Appl. Phys. Lett. 92, 053502 (2008).

${ }^{17}$ D. S. Meyaard, Q. Shan, Q. Dai, J. H. Cho, E. F. Schubert, M. H. Kim, and C. Sone, Appl. Phys. Lett. 99, 041112 (2011).

${ }^{18}$ Q. Dai, Q. Shan, J. Wang, S. Chhajed, J. H. Cho, E. F. Schubert, M. H. Crawford, D. D. Koleske, M. H. Kim, and Y. J. Park, Appl. Phys. Lett. 97, 133507 (2010).

${ }^{19}$ Q. Dai, Q. Shan, J. H. Cho, E. F. Schubert, M. H. Crawford, D. D. Koleske, M. H. Kim, and Y. J. Park, Appl. Phys. Lett. 98, 033506 (2011).

${ }^{20}$ E. F. Schubert, Light-Emitting Diodes, 2nd ed. (Cambridge University Press, Cambridge, 2006). 